\title{
Genetic analyses of pupation distance in Drosophila melanogaster
}

\author{
Marla B. Sokolowski* and \\ Sharon J. Bauer
}

York University, Department of Biology, North York, Ontario M3J 1 P3, Canada.

\begin{abstract}
The inheritance of Drosophila melanogaster larval pupation behaviour is investigated in sixteen reciprocal crosses between field collected lines. These lines were made isogenic for the two major autosomes enabling the data to be analyzed using contrast analysis of variance and biometrical genetic analysis. Results of both analyses showed that the trait "pupation distance", the distance larvae pupate from food, fits a simple additive model of inheritance with no dominance. A chromosomal analysis showed that both the second and third chromosomes act additively on pupation distance and that the third pair of chromosomes had a much larger effect than the second. Significant variability exists in the distance $D$. melanogaster larvae pupate from fruit in nature. This phenotypic variation results from both heritable variation and variation from environmental sources. When the moisture content of the environment surrounding food is modified, gene by environment interactions also contribute to variation in the phenotype. Selective pressures which may act on larval differences in pupation site choice are discussed.
\end{abstract}

\section{INTRODUCTION}

An understanding of the genetic basis of differences in Drosophila larval pupation behaviours is emerging through laboratory and field studies. However, it is difficult to synthesize results since pupation behaviour is measured differently in different assays and, techniques used in genetic analysis of the data are not consistent between studies. In addition, studies which investigate relationships between behavioural differences exhibited in these assays and in fitness are lacking.

The distance a larva pupates from its foraging substrate (pupation distance), is a measure of pupation behaviour most assays have in common. Two early studies of larval pupation behaviour are Sokal et al. (1960) and de Souza et al. (1970). The former used a pupation height assay to measure the distance a $D$. melanogaster larvae pupated from food in vials, while the latter measured the proportion of $D$. willistoni larvae pupated on or off a plug of food in a population cage. The design of most other larval pupation behaviour assays stems from these. Pupation height in vials is measured in Sameoto and Miller (1968), Markow (1979), Ringo

\footnotetext{
* To whom all correspondence should be sent.
}

and Wood (1983), Sokolowski and Hansell (1983), Bauer and Sokolowski $(1985,1988)$, and Casares and Carracedo (1987), in species such as $D$. melanogaster, D. simulans, and D. pseudoobscura, and the tendency to pupate on or off food or fruit is measured by Wong et al., (1985), Sokolowski, (1985), and Godoy-Herrera et al., (in press). In general, genetic analyses have shown that genetic variation for differences in $D$. melanogaster larval pupation behaviour exists in many natural populations, and that the trait can be selected for by artificial selection. Pupation behaviour is polygenically inherited, although, the type of polygenic model suggested in these studies ranges from those having a small number of major genes with many minor modifiers to those with many genes, each having small additive effects. (The former model is most commonly proposed for the on/off food assay, whereas the latter model best describes the pupation height assay results).

In the present study we assay pupation behaviour by measuring "pupation distance". We perform a set of 16 reciprocal crosses between strains differing in pupation behaviour in order to subject the data to contrast analysis of variance and biometrical analysis. Chromosomal analysis is used to determine chromosomal contributions to 
differences in behaviour. The pupation distance assay allows us to measure the distance larvae pupate from a food plug placed on an agar surface in a petri dish. We compare our results with a previous analysis of pupation height which also used crosses between these strains (Bauer and Sokolowski, 1988), and with our ongoing analyses of pupation distance and the tendency to pupate on or off fruit in a field-like assay and in an orchard (Sokolowski, 1985). Our hope is to integrate all of the ongoing studies of larval pupation behaviour and develop a picture of how genes and environment interact, so that in the future we may begin to predict evolutionary outcomes to natural selection at the level of the larval orchard microhabitat.

\section{METHODS}

\section{Strains}

Two strains used in this study are B1B 1 and B15B15, originating from isofemale lines B-1 and B-15 (described in Bauer and Sokolowski, 1984). B1B1 pupates significantly higher in vials than does B15B15 (Bauer and Sokolowski, 1985, 1988). B1B1 and B15B15 (to be called parental strains) were constructed along with B1B15 and B15B1 (chromosomally substituted strains) by using the balanced lethal chromosome technique as described in Bauer and Sokolowski (1985). All four strains are isogenic for chromosomes 2 and 3. Strains are designated with chromosome 2 first and chromosome 3 second. B1B15 and $\mathrm{B} 15 \mathrm{~B} 1$ have chromosome substitutions from B1 B1 and B15B15. For example B1B1 and B1B15 share their second chromosomes (B1), but differ in their third chromosomes.

\section{Crosses}

All 16 reciprocal crosses (as well as their behavioural assays) were performed simultaneously to minimize variation between days known to influence pupation behaviour (Bauer and Sokolowski, 1985, 1988). Flies used in each cross were between 5 and 14 days post-emergence. All animals were maintained under standard conditions $\left(24 \pm 1^{\circ} \mathrm{C}, \mathrm{RH}\right.$ of 60 per cent, uniform overhead illumination and a photocycle of $12: 12$, LD with lights on at $0800 \mathrm{~h}$ ).

\section{Behavioural assay}

The protocol for the pupation distance assay used in this study is described in detail in Wong et al.
(1985). Briefly, petri dishes $(8.5 \mathrm{~cm}$ in diameter and $2.4 \mathrm{~cm}$ high) are filled to a depth of $0.5 \mathrm{~cm}$ with agar $\left(8 \mathrm{~g}\right.$ of agar in $500 \mathrm{ml}$ of $\left.\mathrm{H}_{2} \mathrm{O}\right)$. Subsequently their surfaces are flamed to ensure a smooth surface for larval locomotion. A food plug $(1.8 \mathrm{~cm}$ in diameter and $0.5 \mathrm{~cm}$ high, weighing $1 \cdot 3 \pm 0.05 \mathrm{~g})$ of dead-yeast-agar-sucrose medium initiated with 10 newly hatched 1st instar larvae $( \pm 1.75 \mathrm{~h}$ in age) is placed in the centre of each dish. Each dish is then covered with a lid fitted with two layers of tissue to control condensation. Five replicate dishes were used for each of the 16 reciprocal crosses and the two chromosomally substituted strains (one of the $F_{2}$ crosses had an additional ten replicate dishes). A total of 100 dishes, each with ten larvae, were randomly positioned in the incubator under standard conditions as described above. After the larvae had pupated, we measured the distance that each larva pupated from the food plug and whether the pupa was found on the food plug, on the agar, or elsewhere (on the petri dish wall or lid). Pupae were sexed as in Bauer and Sokolowski (1985).

To test for gene by environment interactions pupation distances of $\mathrm{B} 1 \mathrm{~B} 1$ and $\mathrm{B} 15 \mathrm{~B} 15$ were measured in dishes which differed in the relative water content of their agar base. These were designated WET, MEDIUM and DRY and prepared with 9,18 and 27 grams or agar in one litre of $\mathrm{H}_{2} \mathrm{O}$ respectively. Otherwise, dishes were prepared as described above. Ten replicate dishes were used for each strain for each agar base condition.

\section{Statistical analyses}

For comparison, we use three types of genetic analyses most commonly found in the literature. (1) contrast analysis of variance, (2) biometrical analysis and, (3) chromosomal analysis. To our knowledge, this study is the first to use all three techniques.

We used planned comparisons (Hays, 1981) presented in table 1 to ask questions about the heredity of pupation distance. Pupae were sexed so that male and female pupation distances could be analyzed. The parental contrast compared the pupation behaviour of the progeny of $\mathrm{LL} \times \mathrm{LL}$ to $\mathrm{HH} \times \mathrm{HH}$ where LL represents $\mathrm{B} 15 \mathrm{~B} 15$ and $\mathrm{HH}$ represents B1B1 (see table 2 for a complete description of this nomenclature). The dominance contrast compared parental strains to $F_{1}$ 's. The reciprocal $F_{1}$ contrast compared $F_{1}$ crosses. The Y-chromosome contrast compared male $F_{2}$ progeny. The cytoplasm contrast compared male and female $F_{2}$ progeny. The $X$-chromosome 
Table 1 Contrasts used in the analyses of variance of $D$. melanogaster pupation distance. (Dashes indicate crosses not used in a comparison. See Table 2 for which cross each cross number represents)

\begin{tabular}{|c|c|c|c|c|c|c|c|c|c|c|c|c|c|c|c|c|}
\hline \multirow[b]{3}{*}{ Source } & \multirow{3}{*}{$\frac{\mathrm{LL}}{1}$} & \multirow{3}{*}{$\frac{\mathrm{HH}}{2}$} & \multicolumn{14}{|c|}{ Cross number } \\
\hline & & & \multicolumn{2}{|c|}{$\mathrm{F}_{1}$ 's } & \multicolumn{4}{|l|}{$\mathrm{B}_{\mathrm{L}}$} & \multicolumn{4}{|l|}{$\mathrm{B}_{\mathrm{H}}$} & \multicolumn{4}{|c|}{$F_{2}$ 's } \\
\hline & & & 3 & 4 & 5 & 6 & 7 & 8 & 9 & 10 & 11 & 12 & 13 & 14 & 15 & 16 \\
\hline Parental strains & -1 & +1 & - & - & - & - & - & - & - & - & - & - & - & - & - & - \\
\hline Dominance & -1 & -1 & +1 & +1 & - & - & - & - & - & - & - & - & - & - & - & - \\
\hline Reciprocal $F_{1}$ 's & - & - & -1 & +1 & - & - & - & - & - & - & - & - & - & - & - & - \\
\hline $\begin{array}{l}\text { Y-chromosome } \\
\text { (male data only) }\end{array}$ & - & - & - & - & - & - & - & - & - & - & - & - & +1 & -1 & +1 & -1 \\
\hline $\begin{array}{l}\text { X-chromosome } \\
\text { (female data only) }\end{array}$ & - & - & - & - & +1 & -1 & - & - & +1 & -1 & - & - & - & - & - & - \\
\hline Cytoplasm & - & - & - & - & - & - & - & - & - & - & - & - & -1 & -1 & +1 & +1 \\
\hline $\begin{array}{l}\text { Maternal effects } \\
\text { LL to } F_{1} q\end{array}$ & & & & & & & & & & & & & & & & \\
\hline $\begin{array}{l}\text { LL to } F_{1} q \\
\text { HH to } F_{1} q\end{array}$ & - & - & - & - & +1 & +1 & -1 & -1 & - & - & - & - & - & - & - & - \\
\hline $\begin{array}{l}\text { HH to } F_{1} q \\
\text { Autosomal effects }\end{array}$ & $\overline{-7}$ & $\overline{-7}$ & $\overline{+1}$ & $\overline{+1}$ & $\overline{+1}$ & $\overline{+1}$ & $\overline{+1}$ & $\overline{+1}$ & $\begin{array}{l}+1 \\
+1\end{array}$ & $\begin{array}{l}+1 \\
+1\end{array}$ & $\begin{array}{l}-1 \\
+1\end{array}$ & $\begin{array}{l}-1 \\
+1\end{array}$ & $\overline{+1}$ & $\overline{+1}$ & $\overline{+1}$ & $\overline{+1}$ \\
\hline (all crosses) & & & & & & & & & & & & & & & & \\
\hline
\end{tabular}

contrast compared female backcross progeny. The maternal contrasts were tested using male and female backcross progeny by comparing $L L$ to $F_{1}$ mothers and by comparing $\mathrm{HH}$ to $\mathrm{F}_{1}$ mothers. The autosomal contrast compared all 16 crosses. The expected relationship between the crosses for the autosomal contrast was $\mathrm{LL}<\mathrm{B}_{\mathrm{L}}<\left(\mathrm{F}_{1}=\mathrm{F}_{2}\right)<$ $\mathrm{B}_{\mathrm{H}}<\mathrm{HH}$, this assumes an additive autosomal model with no dominance.

The means, variances, and sample sizes for each of the crosses were used in a biometrical genetic analysis. A weighted least-squares method was used to estimate parameters; the model which best fit the data was then determined by using a goodness-of-fit test (Cavalli, 1952; Mather and Jinks, 1982).

The B1B1, B15B15, B1B15 and B15B1 pupation distance data were subjected to ANOVA to determine whether the second chromosomes, third chromosomes, or second by third chromosome interactions significantly affected pupation distance.

\section{RESULTS}

The low pupating strain B15B15, used in our previous studies pupated closer to food in dishes than did the high pupating strain B1B1. From this we conclude that pupation distance in dishes in these strains is phenotypically correlated to pupation height in vials. The mean pupation distances for each of the 16 reciprocal crosses is given in table 2. No sex difference was found for larval pupation distance, thus the male and female data were pooled. Note however, that a sex difference in pupation height was reported by Bauer (1984), Casares and Carracedo (1987) and Bauer and Sokolowski (1988). This sex difference in pupation height is (1) density dependent, that is, it is only significant when larvae are reared in groups (Bauer and Sokolowski, unpublished) and, (2) only statistically detectable when a large number of strains are analyzed together. A statistically significant sex difference was not found in Bauer and Sokolowski, (1985) because only four strains were compared. A brief glance at the pupation distance data in table 2 shows that the parental strains differ in their pupation distance, the $F_{1}$ progeny are intermediate in phenotype to the parental strains and do not differ from each other, the backcrosses to the low parental line tend to be lower than those to the high parental line, and the $\mathrm{F}_{2}$ progeny show intermediate phenotypes. This gives us our first indication of an additive pattern of inheritance for the trait.

To test this statistically we subject the data to firstly, a contrast analysis of variance (see Wahlsten, 1979; de Belle and Sokolowski, 1987; Bauer and Sokolowski, 1988 for discussions and applications of this technique) secondly, to a biometrical analysis and finally to a chromosomal analysis. An ANOVA is performed on the male and female data separately in order to determine the possible influences of the $\mathrm{X}$ and $\mathrm{Y}$ chromosomes. Table 3 shows the results of the analyses. The only significant contrasts are those which compare the parental lines. Dominance is not sig- 
Table 2 Male and female (pooled) D. melanogaster mean pupation distance in cm (B15B15 with shorter distances is designated LL for low line, B1B1 with farther distances is $\mathrm{HH}$ for high line, $\mathrm{B} 15 \mathrm{~B} 15 \mathrm{q} \times \mathrm{B} 1 \mathrm{~B} 1 \delta^{\circ}$ is $\mathrm{LH}$ and the reciprocal cross is $\mathrm{HL}$ )

\begin{tabular}{|c|c|c|c|c|}
\hline $\begin{array}{l}\text { Cross } \\
\text { number }\end{array}$ & Crosses & $\begin{array}{l}\text { No. of } \\
\text { pupae }\end{array}$ & $\begin{array}{l}\text { Mean pupation } \\
\text { distance }(\mathrm{cm})\end{array}$ & Variance \\
\hline & $\begin{array}{l}\text { Parental Strains } \\
q \times \delta\end{array}$ & & & \\
\hline 1 & $\mathrm{LL} \times \mathbf{L L}$ & 43 & $3 \cdot 58$ & 0.90 \\
\hline 2 & $\begin{array}{l}\mathrm{HH} \times \mathrm{HH} \\
\text { Reciprocal Crosses }\left(F_{1}\right)\end{array}$ & 32 & $4 \cdot 75$ & 0.95 \\
\hline 3 & $\mathrm{LL} \times \mathrm{HH}$ & 46 & $4 \cdot 31$ & $1 \cdot 37$ \\
\hline 4 & $\begin{array}{l}\mathrm{HH} \times \mathrm{LL} \\
\text { Backcrosses } \\
\text { (a) to low line }\left(\mathrm{B}_{\mathrm{L}}\right)\end{array}$ & 38 & $4 \cdot 25$ & $1 \cdot 36$ \\
\hline 5 & $\mathrm{LL} \times \mathrm{LH}$ & 47 & $3 \cdot 70$ & $2 \cdot 05$ \\
\hline 6 & $\mathrm{LL} \times \mathrm{HL}$ & 43 & $4 \cdot 02$ & $1 \cdot 08$ \\
\hline 7 & $\mathrm{LH} \times \mathrm{LL}$ & 48 & 3.78 & $1 \cdot 76$ \\
\hline 8 & $\begin{array}{l}\mathrm{HL} \times \mathrm{LL} \\
\text { (b) to high line }\left(\mathrm{B}_{\mathrm{H}}\right)\end{array}$ & 43 & $3 \cdot 72$ & $1 \cdot 09$ \\
\hline 9 & $\mathrm{HH} \times \mathbf{L H}$ & 39 & $3 \cdot 94$ & $1 \cdot 45$ \\
\hline 10 & $\mathrm{HH} \times \mathrm{HL}$ & 40 & $4 \cdot 26$ & $1 \cdot 68$ \\
\hline 11 & $\mathrm{LH} \times \mathrm{HH}$ & 50 & $4 \cdot 36$ & $1 \cdot 50$ \\
\hline 12 & $\begin{array}{l}\mathrm{HL} \times \mathrm{HH} \\
\text { Reciprocal } \mathrm{F}_{1} \text { Crosses }\left(\mathrm{F}_{2}\right)\end{array}$ & 40 & $4 \cdot 43$ & $1 \cdot 05$ \\
\hline 13 & $\mathrm{LH} \times \mathrm{LH}$ & 130 & $3 \cdot 92$ & $1 \cdot 76$ \\
\hline 14 & $\mathbf{L H} \times \mathrm{HL}$ & 33 & $3 \cdot 68$ & $1 \cdot 60$ \\
\hline 15 & $\mathrm{HL} \times \mathrm{LH}$ & 43 & $3 \cdot 82$ & 1.99 \\
\hline 16 & $\mathrm{HL} \times \mathrm{HL}$ & 43 & $4 \cdot 04$ & $1 \cdot 26$ \\
\hline
\end{tabular}

nificant when the $F_{1}$ progeny are compared to the parental lines and the reciprocal $F_{1}$ progeny do not differ from each other. All non-autosomal factors are not significant. The X-chromosome, Ychromosome, cytoplasmic, and maternal factors do not significantly influence pupation distance

Table 3 Analysis of variance of $D$. melanogaster pupation distance: testing an additive autosomal model with no dominance

\begin{tabular}{|c|c|c|c|}
\hline Source & $\mathrm{df}$ & M.S. & $F$ \\
\hline Model & 15 & $4 \cdot 28$ & \multirow[t]{2}{*}{$2 \cdot 90^{* *}$} \\
\hline Error & 742 & $1 \cdot 48$ & \\
\hline Parental strains & 1 & $25 \cdot 15$ & $17 \cdot 02^{* * *}$ \\
\hline Dominance & 1 & 0.50 & $0 \cdot 34$ \\
\hline Reciprocal $F_{1}$ 's & 1 & $0 \cdot 08$ & 0.05 \\
\hline \multirow{2}{*}{\multicolumn{4}{|c|}{$\begin{array}{l}\text { Y-chromosome } \\
(\text { male data only, } \mathrm{df}=15,322 \text { and M.S.E. }=1 \cdot 42)\end{array}$}} \\
\hline & & & \\
\hline $\begin{array}{l}\text { X-chromosome } \\
\text { (female data onl) }\end{array}$ & $\mathrm{df}=1 \mathrm{1}$ & $\begin{array}{r}1 \cdot 49 \\
\text { and } \mathrm{M} \text {. }\end{array}$ & $\begin{array}{l}0 \cdot 98 \\
1 \cdot 52)\end{array}$ \\
\hline Cytoplasm & 1 & 0.80 & 0.54 \\
\hline \multicolumn{4}{|l|}{ Maternal effects } \\
\hline$\left(\mathrm{LL}\right.$ to $\left.\mathrm{F}_{1} q\right)$ & 1 & 0.51 & $0 \cdot 34$ \\
\hline$\left(\mathrm{HH}\right.$ to $\mathrm{F}_{1}$ ? $)$ & 1 & $3 \cdot 62$ & $2 \cdot 45$ \\
\hline \multicolumn{4}{|l|}{ Autosomal effects } \\
\hline (all crosses) & 1 & $1 \cdot 41$ & 0.95 \\
\hline
\end{tabular}

** $P \leqq 0.001$

*** $P \leqq 0.0001$. and interactions between any and all of these factors are also non-significant.

Biometrical analysis shows that the data fits a simple additive model of inheritance with no dominance. Table 4 shows the parameter estimates of the means, for the midparent value $[\hat{m}]$ and the net additive effect $[\hat{d}]$; these were the only significant genetic parameters. The $t$ values for these parameters and chi-square values for the goodnessof-fit tests are also found in table 4. Heritability of pupation distance as measured in the 16 crosses under the conditions of our experiment is 0.32 as calculated from the variances given in table 4 $[1 / 2 \mathrm{D} /(1 / 2 \mathrm{D}+\mathrm{E})]$.

Table 4 Biometrical analysis showing that the best fit for pupation distance data is a simple additive model with nc dominance. Parameter estimates \pm S.E., $t$ values and goodness. of-fit tests are tabulated

\begin{tabular}{llr}
\hline Parameter & Estimate \pm S.E. & $t$ \\
\hline Means & & 96.43 \\
$\hat{m}$ & $4.05 \pm 0.042$ & 6.22 \\
{$[\hat{d}]$} & $0.51 \pm 0.082$ & \\
\multicolumn{2}{c}{$\chi_{(14)}^{2}=17.08, P=0.25$} \\
Variances & & 9.92 \\
E & $1.17 \pm 0.118$ & 2.68 \\
D & $1.08 \pm 0.403$ \\
& \multicolumn{2}{c}{$\chi_{(14)}^{2}=15.03, P=0.38$} \\
\hline
\end{tabular}


The contrast analysis of variance and biometrical genetic approach have much in common. When all statistical assumptions are met the results of these analyses should be comparable by definition. The biometrical approach uses the data from all of the crosses to simultaneously estimate all of the parameters and test for the best-fitting model. Whereas when we use the contrast analysis of variance the data from only certain crosses are used to test the significance of each effect (table 1). In the present study these approaches gave similar results. The contrast ANOVA showed that pupation distance was inherited autosomally, in an additive fashion with no dominance. The biometrical genetic approach showed that it fitted a simple additive model. In some cases for example when complex maternal effects influence behavioural traits, biometrical analysis and contrast analysis of variance may not arrive at the same conclusions (see Hay, 1972, for a discussion of this problem).

The results of a chromosomal analysis of pupation distance (table 5) shows a significant effect of both the second and third chromosomes but no significant second by third chromosome interaction on differences in this behaviour. The variance component estimates for the second chromosome, third chromosome and interaction were $0.05,0.33$ and 0.00 respectively. The third chromosome had a six to seven-fold greater effect on pupation distance than did the second. While the third chromosome plays a larger role in differences in pupation distance than the second chromosome, the opposite is true for pupation height (Bauer and Sokolowski, 1985).

Table 5 Chromosomal contributions to differences in pupation distances in $D$. melanogaster (males and females pooled)

\begin{tabular}{lrrc}
\hline Source & df & M.S. & \multicolumn{1}{l}{$F$} \\
\hline Model & 3 & $10 \cdot 66$ & $9 \cdot 68^{* * *}$ \\
Error & 146 & $1 \cdot 10$ & \\
2nd chromosome & 1 & 4.81 & $4 \cdot 37^{*}$ \\
3rd chromosome & 1 & $25 \cdot 94$ & $23 \cdot 54^{* * *}$ \\
2nd $\times$ 3rd chromosome & 1 & 1.24 & $1 \cdot 12$ \\
\hline
\end{tabular}

$* P \leqq 0.01$

*** $P \leqq 0.0001$.

Larval pupation behaviour in dishes can also be quantified by measuring the tendency for a larva to leave the petri dish, or, conversely, by measuring the tendency for a larva to pupate on the agar surface (Wong et al., 1985; Sokolowski, 1985;
Godoy-Herrera et al., in press). When we calculate the proportion of larvae which leave each dish for the 16 crosses, arcsin square root transform this data and then subject it to analysis of variance, we find that dish-leaving behaviour also fits a simple additive autosomal model of inheritance. In fact, the major source of variation in pupation distance arises from differences in the distance larvae travel once they have left the dish. We know this because an ANOVA of the 16 crosses for pupation distances of larvae found only on agar was not significant. Only 4 out of 833 pupae were found on the food plug in the present experiment. This low number results from the presence of an agar base surrounding the food plug. When a petri dish contains a food plug which is not surrounded with agar, many more larvae pupate on the food (66/310), however, the strain differences in pupation distance are maintained whether or not the food plug is surrounded by an agar base (Sokolowski, unpublished).

In fig. 1 we see that the pupation distance of $\mathrm{B} 15 \mathrm{~B} 15$ and B1B1 was strongly affected by the relative water content of the agar base. Distances were longest in the WET environment and shortest in the DRY. Similarly, the strain differences

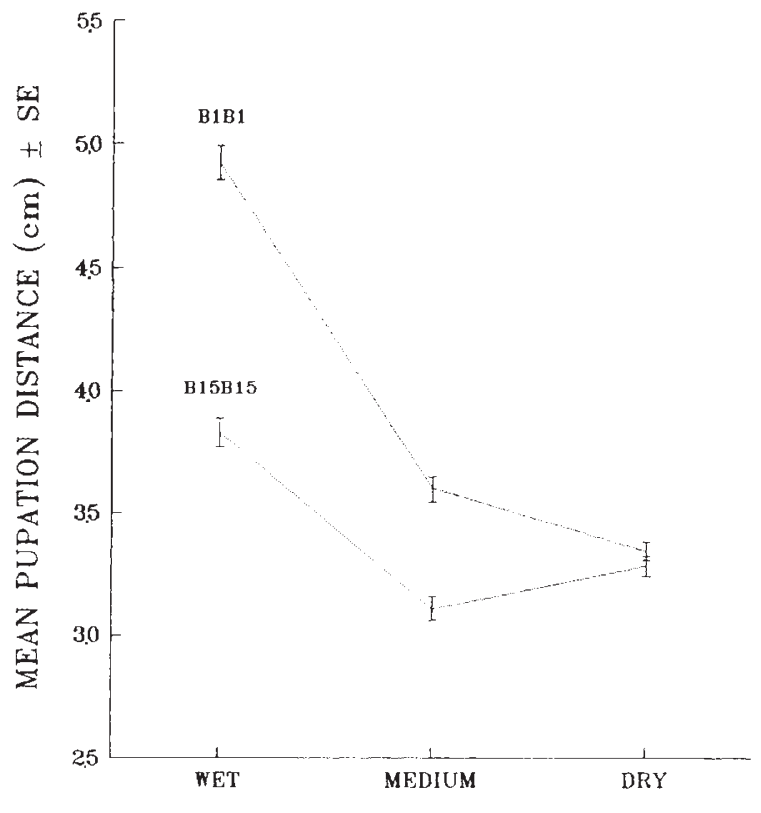

AGAR BASE WATER CONTENT

Figure 1 Norm of reaction curves for the pupation distance of the $\mathrm{B} 1 \mathrm{~B} 1$ and $\mathrm{B} 15 \mathrm{~B} 15$ strains of $D$. melanogaster in dishes whose relative agar base water content varied from wet to dry. 
decreased from being largest in the WET environment to smallest in the DRY environment. Strains respond differently to changes in agar base water content that is, their norms of reaction differed. These patterns were tested in a two-way analysis of variance where the effect of strain $\left(F_{(1,485)}=\right.$ 44.6, $\quad P<0.0001)$, agar base water content $\left(F_{(2,485)}=66.9, P<0.0001\right)$ and their interaction $\left(F_{(2,485)}=12.6, \quad P<0.0001\right)$ were all highly significant.

\section{DISCUSSION}

What do most pupation behaviour assays have in common and what, if any, generalizations can be drawn from the pupation behaviour data? Strain differences in the tendency of larvae to move away from moist larval substrates to pupate are shown whether the substrate is a food plug on a plastic petri dish bottom, a yeast plug on an agar base in a petri dish bottom, food in the bottom of a vial, or fruit on soil (Sokolowski, 1985). The genetic basis to differences in pupation behaviour in all of these assays is autosomal with little or no dominance. The relative contributions of the second as compared to the third pair of autosomes is however, dependent on the pupation behaviour of interest. For example, pupation height in vials has a greater second chromosome contribution (Bauer and Sokolowski, 1985) whereas pupation distance in dishes (present study) and in a field assay (Sokolowski, unpublished) has a greater third chromosome contribution. This difference in chromosomal contribution may result from the third chromosome making a greater contribution to pupation behaviours with horizontal as opposed to vertical locomotory movement (i.e., the pupation height measure may have a geotactic component that is influenced by second chromosome genes). Despite these differences in proportional chromosome contribution, we have found that most laboratory stocks, isofemale lines and, field strains collected from different pupal microhabitats, show a correlation in pupation behaviour which can be described on the basis of a distance measure, that is, how far in any direction a larva pupates from the larval feeding substrate.

Indeed, strains showing high pupal heights pupate farther from the food in dishes, field-like assays, and orchard microhabitats and, when the on/off fruit measure is considered, they also pupate more off-food. In contrast, low-pupating strains pupate on or near the food in dishes and in field-like assays, and more on the food in our orchard field site (Sokolowski, 1985). This correlation enables us to predict that as a consequence of selection on one trait (for example pupation height) we should expect a correlated response in others of these traits (for example on/off food). The ability to select on both traits simultaneously should provide us with a more detailed indication of the degree of genetic correlation between these behavioural traits. Of further interest is whether the pattern of polygenic inheritance for pupation behaviours is a classical additive one [as is suggested for pupation height (Bauer and Sokolowski, 1988) and pupation distance (this paper)] or one consisting of several major genes with many minor modifiers [as is suggested for the on/off food measures (de Souza et al., 1970; Godoy-Herrera et al., in press)].

Of primary concern to any investigation of the interrelationships between behaviour, ecology, genetics and changes in these factors is the identification of potential selective pressures which may act on behavioural differences. For the case of pupation behaviour these include (1) density dependent selection, (2) selection resulting from dessication in dry environments or putrification of pupae in very moist environments, and (3) pupal parasitoids of Drosophila. An example of the first is given in Mueller and Sweet (1986), who show that when larvae are reared at high densities, pupation sites away from food are selected for, possibly because pupae found on the food (low in vials) are drowned by the activity of a high density of foraging third instar larvae. An example of the second is given in Sokolowski (1985), who shows that larvae which pupate on-fruit have greater pupal survivorship than those found off-fruit when the soil surrounding the fruit is dessicated. However, when soil and fruit are wet, larvae found pupating on-fruit are more susceptible to rot than those pupating on or in soil. Most larvae show some degree of behavioural plasticity in response to variation in moisture in the laboratory and field environments. As water content increases so does the distance larvae pupate from the food. Drosophila pupal parasitoids, such as the pteromalid Pachycrepoideus which are found in our orchard field site, represent a third selective pressure which may differentially act on pupae found in different microhabitats (for example on/off fruit). Much work needs to be done with the aim of relating genetically determined behavioural differences to differences in fitness. 
Acknowledgements The authors thank M. Collins and T. Tully for performing the biometrical genetic analysis, J. S. de Belle, L. Rodriguez and L. Chow for technical assistance, A. Shaw, D. E. MacDonald, and J. S. de Belle for comments on a preliminary manuscript, J. S. de Belle for drawing fig. 1, and A. Varosi for expertly typing the manuscript. The work was supported by a Natural Sciences and Engineering Council of Canada Operating Grant No. A3397 to M.B.S.

\section{REFERENCES}

BAUER, S. J. 1984. Sex differences in pupation site choice in Drosophila melanogaster. Dros. Inf. Serv., 60, 58.

BAUER, S. J. AND SOKOLOWSKI, M. B. 1984. Larval foraging in isofemale lines of Drosophila melanogaster and $D$. pseudoobscura. J. Heredity, 75, 131-134

BAUER, S. J. AND SOKOLOWSKI, M. B. 1985. A genetic analysis of path length and pupation height in a natural population of Drosophila melanogaster. Can. J. Genet. Cytol., 27, 334340 .

BAUER, S. J. AND SOKOLOWSKI, M. B. 1988. Autosomal and maternal effects on pupation behaviour in Drosophila melanogaster. Behav. Genet., 18, 81-97.

CASARES, P. AND CARRACEDO, M. C. 1987. Pupation height in Drosophila: Sex differences and influences of larval developmental time. Behav. Genet., 17, 523-535.

CAVALLI, L. L. 1952. An analysis of linkage in quantitative inheritance. In Reeve, E. C. R., and Waddington, C. H. (eds) Quantitative Inheritance London, HMSO, pp. 135144.

DE BELLE, J. S. AND SOKOLOWSKI, M. B. 1987. Heredity of rover/sitter: Alternative foraging strategies of Drosophila melanogaster. Heredity, 59, 73-83.

DE SOUZA, H., DA CUNHA, A. AND DOS SANTOS, I. 1970. Adaptive polymorphism of behavior evolved in laboratory populations of Drosophila willistoni. Am. Nat., 104, 175189.
GODOY-HERRERA, R., CIFUENTES, L., DIAZ DE ARCAYA, M. F., FERNANDEZ, M., FUENTES, M., REYES, I. AND VALDERRAMA, C. The behaviour of Drosophila melano gaster larvae during pupation. Anim. Beh. (in press).

HAY, D. A. 1972. Genetical and maternal determinants of the activity and preening behaviour of Drosophila melanogaster reared in different environments. Heredity, 28, 311-336.

HAYS, W. L. 1981 Statistics, 3rd ed. Holt, Rinehart and Winston, New York.

MARKOW, T. A. 1979. A survey of intra- and interspecific variation for pupation height in Drosophila. Behav. Genet., 9, 209-217.

MATHER, K. AND JinkS, J. L. 1982. Biometrical Genetics. Chapman and Hall, New York.

MUELleR, L. D. AND SWEET, V. F. 1986. Density-dependent natural selection in Drosophila: Evolution of pupation height. Evolution, 40, 1354-1356.

RINGO, J. AND WOOD, D. 1983. Pupation site selection in Drosophila simulans. Behav. Genet., 13, 17-27.

SAMEOTO, D. AND MILlER, R, 1968. Selection of pupation site by Drosophila melanogaster and D. simulans. Ecology, 49, 177-180.

SOKAL, R., EHRLICH, P., HUNTER, P. AND SCHLAGER, G. 1960. Some factors affecting pupation site of Drosophila. Ann. Entom. Soc. Am., 53, 174-182.

SOKOLOWSKI, M. B. 1985. Ecology, genetics and behaviour of Drosophila larval foraging and pupation behaviour. $J$. Insect Physiol., 31, 857-864.

SOKOLOWSKI, M. B. AND HANSELL, R. I. C. 1983. Elucidating the behavioral phenotype of $D$. melanogaster larvae: Corre. lations between foraging strategies and pupation heights. Behav. Genet., 13, 267-280.

WAHLSTEN, D. 1979. A critique of the concepts of heritability and heredity in behavioural genetics. In Royce, J. R. and Mos, L. (eds) Theoretical Advances in Behavioural Genetics, Sijthoff and Nordhoff, Germantown, MD, pp. 425-481.

WONG, J. L., SOKOlowski, M. B. AND KENT, C. F. 1985. Prepupation behaviour in Drosophila: Embedding. Behav. Genet., 15, 155-164. 